\title{
École, multimédia et Internet
}

\section{Guy Pouzard}

\section{(2) OpenEdition}

Journals

Édition électronique

URL : http://journals.openedition.org/ries/2894

DOI : 10.4000/ries.2894

ISSN : 2261-4265

\section{Éditeur}

Centre international d'études pédagogiques

\section{Édition imprimée}

Date de publication : 1 juin 1998

Pagination : 25-35

ISSN : 1254-4590

\section{Référence électronique}

Guy Pouzard, «École, multimédia et Internet », Revue internationale d'éducation de Sèvres [En ligne], 18 | juin 1998, mis en ligne le 18 avril 2013, consulté le 01 mai 2019. URL : http:// journals.openedition.org/ries/2894; DOI : 10.4000/ries.2894

Ce document a été généré automatiquement le 1 mai 2019.

(c) Tous droits réservés 


\title{
École, multimédia et Internet
}

\author{
Guy Pouzard
}

\section{La situation générale}

\section{Une école profondément concernée}

1 L'école, dans ses missions de préparation à l'épanouissement de la personne comme dans celles d'insertion sociale et professionnelle ne peut (ni ne doit) se tenir à l'écart des profonds bouleversements qui modifient les métiers, changent la nature et l'organisation du travail, remodèlent les mentalités et les rapports entre les individus. Élément essentiel de l'égalité des chances, elle doit offrir à tous les mêmes possibilités de préparation aux nouvelles formes de travail et d'accès aux nouveaux supports de la connaissance, du savoir ou de l'information. Comme elle l'a fait à l'époque du développement de la société industrielle pour des raisons de méthode (Guizot avec la généralisation de l'enseignement simultané rendu obligatoire en 1830 après un arrêté royal de 1818) ou pour des raisons sociales et politiques (J. Ferry avec l'obligation scolaire et la gratuité pour tous en 1885), elle doit impérativement réfléchir à ses propres structures et ses propres méthodes pour assurer dans les meilleures conditions possibles ses missions avec les meilleures chances de succès dans une société mouvante. Les investissements et les enjeux colossaux que représentent les NTIC ne peuvent se faire sans une optimisation de leur utilisation, sans une définition nouvelle des objectifs de l'école, sans mettre en place des structures, des méthodes pédagogiques et des formations adaptées. De la même façon que le XXI ${ }^{e}$ siècle a vu se mettre en place les méthodes de l'enseignement simultané gratuit et obligatoire dans la classe, le $\mathrm{XXI}^{\text {e }}$ siècle devra mettre en place de nouvelles méthodes d'enseignement, de nouvelles stratégies éducatives s'appuyant sur le rôle essentiel que jouent les NTIC dans la société de l'information et sur les méthodes éducatives nouvelles qu'elles sont susceptibles d'apporter (travail coopératif sur les réseaux, mutualisation des ressources, partage de l'intelligence, etc.).

2 L'enseignement technique et l'enseignement professionnel ne sont plus les seuls utilisateurs directement concernés par l'utilisation de techniques en évolution rapide. 
L'outil multimédia, que nous prenons dans le sens « numérique » c'est-à-dire passant par un micro-ordinateur communiquant, est utilisable par tous, quel que soit l'âge, quelle que soit la discipline, quelle que soit la place dans le système éducatif. De plus, le microordinateur de l'école est en tout point semblable au micro-ordinateur utilisé aujourd'hui dans la majorité des postes de travail des entreprises. Cette situation est nouvelle. Si on a longtemps estimé que seuls les enseignements techniques et professionnels devaient être dotés d'outils se rapprochant au mieux de ce que les élèves allaient utiliser dans l'exercice de leur profession future, aujourd'hui, tous les élèves sont concernés.

\section{Une situation contrastée}

Pourtant, de grandes différences se rencontrent dans tous les ordres d'enseignement: entre les écoles d'une même ville, entre les collèges d'un même département, entre les lycées d'une même région, avec de grandes inégalités quant à la présence et à l'utilisation de l'ingénierie éducative. Il s'agit souvent de différences dues à l'attitude des collectivités territoriales compétentes, à l'implication des chefs d'établissement ou à la préparation des enseignants.

Or l'informatique pédagogique se doit d'être accessible à tous les élèves comme à tous les enseignants. Elle doit surtout privilégier la nature didactique des contenus. De ce fait, il est nécessaire d'avoir une politique cohérente pour développer une informatique souple, diversifiée, conviviale, non réservée à des spécialistes et disponible partout. En plus des fonctions traditionnelles (traitement de textes, tableurs, grapheurs...), elle doit autoriser la saisie et le traitement des données diverses en temps réel (textes, fichiers, images, son...). Elle n'a pas uniquement un caractère informatif. Elle implique en toute hypothèse l'accès aux réseaux (intranets ou internet), pour un grand nombre d'utilisateurs avec des débits convenables et des coûts raisonnables. L'élève comme l'enseignant doivent être actifs autant qu'interactifs, consommateurs autant que créateurs. Les futurs appareils, légers et faiblement encombrants que l'on peut qualifier de "nomades ", mais dotés de grandes capacités de communication risquent bien de modifier encore davantage les situations pédagogiques.

\section{Dans les classes}

\section{Des bases classiques}

On se demande trop rarement si l'organisation de la classe dans sa forme classique est la forme la plus adaptée pour un rendement optimal de l'utilisation par les élèves des outils multimédia, et les réflexions sur les possibilités d'améliorations du système se font pratiquement toujours dans le cadre des méthodes de l'enseignement simultané. On admet comme une évidence que les nouvelles technologies doivent être au service des disciplines dans une classe traditionnelle dont la structure, destinée à optimiser le rendement du système éducatif, provient pourtant d'une époque où les technologies de l'éducation étaient limitées à la transmission du savoir par la parole du maitre et le tableau noir.

6 Aujourd'hui, on trouve des systèmes sophistiqués de visioconférence pour que des élèves puissent, par exemple, bénéficier d'enseignement d'options ou de langues rares. L'utilisation qui est faite consiste essentiellement à « transporter » par l'intermédiaire du 
réseau numérique un enseignement dont la forme reste analogue à celle de l'enseignement dispensé dans une classe traditionnelle. Il s'agit plus de la mise en place d'une forme "locale» de télévision scolaire que de l'exploitation des différentes possibilités d'un réseau interactif. Dans certains cas, devoirs et corrections sont même encore envoyés par la poste.

Les enseignants impliqués dans l'utilisation de l'outil multimédia agissent la plupart du temps individuellement, en fonction d'un matériel qu'ils n'ont souvent pas pu choisir. Les développements sont pratiquement toujours le résultat de la présence dans un établissement d'un ou plusieurs professeurs passionnés, novateurs et, dans bien des cas, formés à la suite du plan « informatique pour tous » lancé en 1985.

Rares sont encore les établissements qui mènent une politique de projet en matière d'information et d'utilisation de l'outil multimédia. Celle-ci est souvent l'affaire du seul $\mathrm{CDI}$, aux fonctions très diversement comprises et qui utilise rarement le réseau comme source pour rechercher une information pertinente nécessaire à la pédagogie.

On peut souligner néanmoins que l'enseignement privé catholique sous contrat a mis en place depuis la rentrée scolaire 1996 un accord de partenariat avec un grand constructeur et développé un réseau pédagogique (Scolanet) utilisé par l'ensemble de ses établissements. Les enseignants reçoivent tous une formation de base nécessaire pour l'utilisation des outils et du réseau.

\section{De nombreux exemples d'utilisation}

10 On trouve des exemples d'utilisation de l'outil multimédia dès la classe maternelle. Dans l'enseignement primaire, ils correspondent à diverses situations d'apprentissages fondamentaux pour lesquelles l'apport du son, de l'image et de séquences animées est un atout important. Les enfants montrent presque sans exception intérêt et aptitudes. De bons résultats sont rapidement obtenus en lecture et en écriture. Il est indispensable de souligner l'importance toute spéciale qu'il faut accorder à l'ergonomie des conditions de travail chez les enfants. On doit la retrouver dans l'interface enfant/machine, mais aussi dans le mobilier, l'éclairage, l'organisation de l'espace et du temps.

11 On avait vu se développer au cours de ces dix dernières années des formes d'écriture collective utilisant les réseaux de classe (cependant, seules 120 classes environ participaient au réseau en 1992). Même si rares sont encore les classes disposant d'un matériel multimédia suffisant, en quantité comme en qualité, la production de journaux d'écoles, parfois d'une étonnante qualité, constitue une très grande partie de l'utilisation de l'outil multimédia dans l'enseignement primaire. On assiste à l'éclosion de très nombreux journaux dont beaucoup se trouvent sur Internet, forme moderne de l'imprimerie chère à $C$. Freinet. Si l'idée de pédagogie active n'a pas attendu l'existence du multimédia pour apparaître, son développement peut être grandement facilité par cet outil (il faut insister sur le fait que l'utilisation de l'outil multimédia n'a pas à être permanente : dans les classes qui aujourd'hui l'utilisent le mieux, il y a un temps pour des activités avec le micro-ordinateur et un temps pour d'autres formes de travail plus classiques).

Dans les collèges, comme dans les écoles, la présence d'un enseignant compétent, dévoué et très impliqué a toujours été un élément déterminant. L'effet d'entraînement est indéniable et des collèges isolés peuvent devenir des pôles d'expérimentations variées 
dans diverses disciplines. Très souvent, c'est à partir de projets de type «projet d'action éducative » que sont développées des réalisations pluridisciplinaires ou touchant à la vie scolaire. Si l'utilisation de l'outil multimédia s'intègre très bien dans le cadre de projets, de nombreux enseignants soulignent la difficulté de l'utilisation de ces outils dans le cadre de la classe traditionnelle. La maîtrise des matériels mais aussi la rigidité des emplois du temps, les changements de classes, sont des facteurs essentiels qui accroissent les difficultés.

13 Au lycée, la situation est encore différente. Dans les enseignements technologiques et professionnels, l'utilisation de l'informatique est généralisée, bien plus d'ailleurs que celle du multimédia. Cependant l'informatique intervient davantage dans la perspective d'une situation professionnelle future que comme outil pédagogique. Les logiciels utilisés sont spécifiques à la discipline (logiciels de comptabilité, de commande numérique, etc.). Certains professeurs utilisent néanmoins avec bonheur les ressources du multimédia pour un enseignement disciplinaire personnel, vivant, attractif et interactif. S'il existe dans quelques cas, le travail en réseau n'est cependant pas la règle générale. L'outil informatique est souvent utilisé par les élèves pour la présentation de leurs travaux personnels (traitements de texte et tableurs).

Dans l'enseignement général, les professeurs soulignent ici aussi l'inadéquation entre multimédia, programmes et horaires. Les réalisations de qualité ont presque toujours été initialement développées dans le cadre des ateliers de pratiques des techniques d'information et de communication (APTIC), en particulier dans les disciplines artistiques. Des enseignants ont parfaitement assimilé tout ce que pouvait apporter le traitement du son dans l'enseignement de la musique. Leurs cours, par leur originalité et leur richesse, suscitent un intérêt évident chez les élèves.

Les disciplines scientifiques montrent toutes des exemples d'application, que ce soit en mathématiques, en physique, en chimie, en géologie ou en biologie. Plusieurs des possibilités offertes par l'informatique sont d'ailleurs mises à profit, qu'il s'agisse de l'illustration du cours par l'intégration d'images, de séquences vidéos, de simulations ou d'expérimentations assistées par ordinateur, ces dernières étant mises en œuvre depuis déjà plusieurs années en biologie.

16 L'outil multimédia n'est pas limité au seul domaine des enseignements scientifiques. Des utilisations intéressantes montrent les possibilités de l'hypertexte en littérature pour la syntaxe, la grammaire, l'écriture, la recherche documentaire, etc. L'enregistrement et la restitution immédiate de la voix et son intégration dans des documents hypermédia font également du multimédia un outil de choix pour aider à l'apprentissage des langues vivantes, d'Internet un outil d'étude des civilisations. Il en va de même pour l'enseignement des arts plastiques ou de l'histoire de l'art. La possibilité d'intégration de séquences vidéo dans des documents hypermédia se retrouve également en éducation physique et sportive où elles servent à illustrer les règles ou les phases d'un jeu d'équipe, à décomposer et analyser les mouvements d'un sport individuel.

17 Que ce soit dans l'enseignement primaire, au collège ou au lycée, des expériences conduites avec des élèves en difficulté dans des environnements réputés difficiles montrent l'impact positif de l'utilisation de l'outil multimédia sur leur comportement qui, de l'avis des professeurs, s'améliore notablement. De même, son utilisation offre d'intéressantes possibilités pour des élèves hospitalisés ou pour des élèves amenés à être souvent hors de la classe traditionnelle comme les sportifs de haut niveau. Elle présente, enfin, un grand intérêt pour des élèves en difficulté scolaire. En valorisant leur travail, le 
micro-ordinateur leur redonne le goût de l'effort et du travail bien fait. D'une façon plus générale, le comportement des élèves qui se sentent plus responsables et soucieux de la qualité du travail réalisé quand ils ont l'occasion de travailler avec des outils multimédia, est amélioré. On pourrait penser que cet aspect ne concerne que certains élèves privilégiés mais l'utilisation des technologies multimédia avec des élèves en difficulté ou dans des environnements difficiles montre qu'il s'agit bien d'une règle générale. La satisfaction des enseignants est également réelle, malgré les difficultés souvent soulignées.

18 Tous les exemples montrent que l'usage de l'outil multimédia est peu compatible avec des séquences horaires trop courtes, des emplois du temps trop rigides, des changements de classe trop fréquents. Dans ce cas, seule une maîtrise parfaite de l'outil peut permettre d'envisager son utilisation dans la classe de conception traditionnelle. Cette situation peut expliquer bien des réticences et le report sur le CDI de la responsabilité de l'utilisation des nouvelles technologies. Elle est dangereuse car elle marginalise le multimédia, charge le CDI de l'essentiel des responsabilités, confine trop souvent l'outil dans le rôle particulier de préparation et d'édition d'un journal d'établissement et ralentit ou empêche son appropriation par les enseignants.

\section{La révélation du vecteur Internet}

Les réseaux télématiques ont parfois été exploités assez tôt dans l'enseignement primaire (avec le Minitel par exemple), ainsi que dans différentes disciplines comme le français, l'histoire et géographie, les sciences et bien sûr dans les langues vivantes. Ce type d'utilisation soulève le problème des normalisations nécessaires et de l'ergonomie des systèmes (protocoles et interfaces de communication, jeux de caractères, codage, décodage, convivialité, etc.) pour assurer la cohérence des communications, les rendre intelligibles et utilisables.

\section{Vers une affirmation des usages pédagogiques}

Si l'utilisation du réseau Internet demande un minimum de moyens adaptés et de connaissances en matière de communications numériques, les élèves, dès l'école élémentaire, montrent de réelles dispositions pour ce type de travail. Les pages qu'ils créent sur le Web peuvent témoigner d'indéniables qualités de recherche, d'imagination et de sérieux. Certaines écoles à classe unique (en particulier celles du réseau Freinet) ont ainsi acquis une notoriété nationale et internationale par la qualité du travail présenté sur Internet. Leurs sites sont souvent plus connus de l'étranger que de la France même si plusieurs articles de presse ou émissions de télévision leur ont déjà été consacrés.

21 Les expériences, généralement antérieures à la mise en place d'actions nationales, d'utilisation du courrier électronique par des classes dans différentes disciplines, notamment en français et en langues étrangères sont relativement nombreuses. Les échanges internationaux se multiplient, ceux qui semblent fonctionner le mieux étant de type bilatéral. L'écriture et la mise en forme des «pages Web» ainsi que les échanges linguistiques constituent souvent la partie la plus importante du travail. L'intérêt des écoles étrangères, francophones ou non, se manifeste dans les échanges de courrier, de messages ou des demandes de coopération. Les établissements français d'enseignement établis à l'étranger commencent eux aussi à utiliser activement Internet. 

diffusion consacrées à l'éducation augmente sans cesse. Sans être exhaustif, on peut citer entre autres Rescol, CDIDOC, Isoc-educ, ntic-colleges. De nombreux enseignants créent leur site, des forums sont ouverts à l'occasion des consultations nationales. Une utilisation plus extensive des possibilités offertes par le réseau en matière de ressources informatives et de travail coopératif, avec aussi des outils de type workflow utilisant les ressources d'Internet, deviendra sans doute indispensable dans une étape ultérieure.

L'utilisation d'Internet au lycée d'enseignement général reste moins développée que celle qui en est faite à l'école ou au collège. Sauf dans quelques établissements expérimentaux, elle est, pour l'instant, essentiellement limitée à la mise à disposition sur le réseau d'informations concernant le lycée, les formations qui y sont dispensées ainsi que les orientations pédagogiques contenues dans le projet d'établissement.

\section{Des difficultés réelles}

Si des changements importants ont pu être observés là où les " précurseurs » se montrent actifs, ils ne doivent pas être l'arbre qui cache la forêt. D'importantes difficultés subsistent en effet, notamment pour étendre les utilisations au plus grand nombre. Elles peuvent avoir plusieurs origines, tant matérielles que psychologiques, fonctionnelles que structurelles. Elles sont liées, entre autres, aux emplois du temps, aux matériels, aux conditions d'accès aux réseaux, à la maintenance, aux programmes ou aux examens, mais aussi, trop souvent encore, à l'absence de prise en compte par le système lui-même des efforts consentis, des progrès réalisés, des changements dans les méthodes de travail, dans les approches pédagogiques ou dans la nécessité de formation. L'action de responsables locaux, parfois loin d'être convaincus de l'utilité même de l'utilisation du multimédia et encore plus des réseaux, peut conduire au désenchantement, à la lassitude ou, pire, au découragement. Quelques exemples d'évaluation montrent la grande différence d'approche, dans l'utilisation du réseau, par exemple, entre les enseignants utilisateurs et les évaluateurs.

\section{Des demandes cependant insistantes}

Très nombreux sont les élèves qui souhaitent pouvoir accéder au réseau Internet et travailler à la mise en place de pages Web pour leur école, leur collège ou leur lycée. Dans tous les cas, enseignants et élèves qui utilisent le multimédia, en ligne ou hors ligne, ne sont pas avares de leur temps de travail.

Sensibilisées par les innombrables pages des journaux ou les rubriques spécialisées des radios et des chaînes de télévision comme par l'utilisation sans cesse croissante dans la vie quotidienne (vente par correspondance, nouveaux postes de travail...), les familles, dont l'attente est grande, posent la question de l'accès au multimédia d'une façon presque systématique.

De nombreuses associations, parfois créées par les collectivités locales, offrent un accès régulier à du matériel multimédia et des possibilités de formation pour les jeunes comme pour les adultes. Des municipalités ont mis en place des « cyber espaces » qui connaissent un grand succès auprès des jeunes. Certaines associations périscolaires jouent un rôle très positif en termes de développement d'activités liées au multimédia comme en termes de réflexion sur leurs usages. Des enseignants y ont un rôle déterminant. 

incessantes modifications technologiques.

\section{Une réflexion pédagogique à mener} tranquille». ses investissements. utilisation des outils multimédia reste une nécessité.

Des entreprises qui ont de plus en plus besoin de personnes qualifiées ou, plus encore, ayant une véritable culture d'utilisation des outils multimédia, sont également soucieuses de la formation donnée par l'école dans ce domaine. Cette formation de base est d'ailleurs indispensable pour permettre à chacun de pouvoir ensuite bénéficier pleinement de l'indispensable formation continue et ne pas se trouver totalement démuni devant les

Les collectivités territoriales enfin sont soucieuses de prendre en compte les nécessités sociales et les aspirations des habitants, qu'ils soient dans des quartiers difficiles ou dans des régions isolées. Leur rôle dans le développement des réseaux est essentiel.

Dans les établissements, l'introduction du multimédia numérique et l'utilisation des réseaux doit conduire à une réflexion de fond sur les pratiques éducatives des enseignants et sur le rôle du CDI. Celui-ci doit devenir le centre nerveux de l'information et ne pas être cantonné dans un rôle purement documentaire ou d'espace de «lecture

31 Les technologies de communication et d'information, comme le travail sur réseaux s'accommodent mal de découpages horaires stricts et du manque de souplesse issus directement de la forme traditionnelle de «l'enseignement simultané ». Aussi paradoxal que cela puisse paraitre au premier abord, elles représentent une forme de travail beaucoup plus personnalisée et collective à la fois. Il n'est pas évident que l'expression « ordinateur dans la classe » - si on entend par «classe » la classe traditionnelle et son organisation - représente la forme optimale de l'utilisation de l'ingénierie éducative et de

Les exemples observés montrent en effet que les meilleures réalisations sont issues de pratiques pédagogiques dans lesquelles l'utilisation du temps est souple et qui s'écartent notablement de celles de la salle de classe traditionnelle. On vérifie dans les faits que l'utilisation des technologies éducatives est plus productive à l'école primaire, y compris dans les classes maternelles où elle a pu être observée. Il s'agit de situations où le maitre ne change pas et où l'organisation de l'emploi du temps de la classe peut être plus souple qu'au collège et plus encore qu'au lycée. Même si, de leur côté, les enseignements techniques et professionnels ont considérablement fait évoluer leurs formes pédagogiques, la recherche-développement dans la perspective d'une meilleure

L'introduction de leur utilisation sous forme explicite dans les programmes de l'enseignement primaire, même si l'accent est surtout mis sur l'aspect informatique, est un pas important. À l'autre extrémité, l'introduction d'une option informatique en classe de seconde et en classe de première ne reflète pas les nécessaires continuité et progressivité des apprentissages. En outre, enseignants et élèves sont tenus, surtout dans les classes terminales, par le programme et surtout par l'examen final.

4 Si une réflexion sur la place du multimédia tout au long du cursus est nécessaire, c'est évidemment le plus tôt possible qu'il faut commencer à utiliser et à comprendre le rôle comme le fonctionnement des ces technologies éducatives par des apprentissages progressifs et raisonnés. En l'état actuel des choses, il convient de généraliser 
l'introduction de l'utilisation des technologies multimédia dans les programmes et dans les examens, tout en étant conscient de l'importance des exigences que cela entraîne.

Les élèves peuvent commencer très tôt cet apprentissage de façon à acquérir progressivement un socle commun de connaissances et de pratiques relatif aux principes généraux de fonctionnement de l'outil multimédia, qui leur permette d'aller au-delà d'une utilisation purement instrumentale. Ce noyau de connaissances ne constitue pas un préalable à l'utilisation mais doit permettre d'éclairer l'usage pertinent et raisonné des technologies numériques dans lequel l'école trouve tout son sens. Il s'agit en effet d'accéder à la maîtrise intellectuelle et pratique des outils de communication et à la découverte des nouveaux métiers qu'ils engendrent. Il doit ainsi contribuer au développement de la connaissance et des savoir-faire nécessaires à l'épanouissement des potentialités de chacun pour en faire un citoyen actif et responsable.

L'outil ne doit pas commander la pédagogie. Il doit donc être lui-même parfaitement maîtrisé, pour que le système éducatif sache tirer des technologies numériques tous les avantages et bénéfices nouveaux en matière de créativité, de responsabilisation des individus, de travail en équipe et de partage de l'intelligence. Ce n'est pas uniquement comme outil de «navigation » qu'il faut concevoir l'usage des réseaux. C'est bien (il faut s'en persuader!) un élément important de la société, un outil de travail indispensable. Dans l'état actuel du système éducatif, l'utilisation doit en être envisagée d'abord dans les programmes, mais aussi à terme dans les examens ou concours.

Les changements fondamentaux de la société exigent une adaptation en profondeur du système éducatif. Il s'agit d'un investissement nécessaire pour l'avenir dont le retour sera productif mais dont l'absence serait un préjudice.

38 L'impact des technologies d'information et de communication sur l'école et sur ses liens avec la société doit être un objet d'étude et de réflexion. Il faut aussi examiner leurs conséquences sur les nouvelles formes de travail, plus personnelles et plus collectives dans le même temps, que va développer l'usage généralisé des réseaux. Il faut donc également prendre en compte par une analyse sociologique et philosophique les nouvelles formes culturelles dans lesquelles les environnements numériques et virtuels joueront un rôle de plus en plus grand.

\section{Former les personnels}

39 La formation initiale et continue des enseignants doit impérativement prendre en compte l'utilisation et la compréhension de techniques qui constituent un élément indispensable de la société de l'information. La rapidité de l'évolution des technologies exclut les formations trop ciblées qui risquent d'être dépassées avant même d'avoir été mises en place. Les instituts de formation des maitres, les missions académiques à la formation des personnels, comme les instances de formation des personnels d'encadrement, doivent intégrer cette nouvelle dimension de la pédagogie. Les référentiels de compétences des enseignants et donc les programmes de formation et de contrôle dans les instituts universitaires de formation des maitres, doivent inclure, comme cela se fait dans d'autres pays, les aptitudes à utiliser l'outil multimédia à des fins pédagogiques, aussi bien pour les utilisations « en ligne » que pour les utilisations « hors ligne ». Un minimum de formation générale pour tous est indispensable si l'on veut que les usages se multiplient et le pragmatisme doit guider les actions de formation. La préparation des enseignements, la 
réflexion sur les méthodes, le travail lui-même doivent être conçus sur les réseaux et de manière interactive.

Les enseignants ne sont pas seuls concernés. Les personnels de direction et d'inspection ont à jouer un rôle essentiel dans la prise en compte des outils d'information et de documentation, aussi bien pour les aspects pédagogiques que pour la vie scolaire en général. De nouvelles compétences et de nouveaux métiers doivent être aussi définis pour les personnels non enseignants qui auront à participer à la maintenance et à veiller au bon fonctionnement des réseaux, des matériels ou des logiciels. Le fonctionnement d'un réseau se gère et se surveille par le réseau.

\section{Matériels et réseaux}

41 Les accès et les équipements correspondants doivent être disponibles à chacun des niveaux des partenaires institutionnels publics (État, collectivités territoriales, établissements publics) ou privés. Il s'agit en fait d'une véritable politique d'aménagement du territoire, qui demande à être identifiée dans des cahiers des charges clairement définis pour tous les établissements scolaires qui doivent pouvoir entrer dans un cadre d'objectifs communs définis sur l'ensemble du territoire, de façon à éviter des répartitions trop inégalitaires.

La généralisation de l'utilisation des réseaux nécessitant des débits élevés implique de nouvelles stratégies d'accès qui requièrent une réflexion globale. Dans le cadre de cette réflexion, la rapide obsolescence des matériels doit être prise en compte ainsi que la diversité de leurs applications. Les recommandations issues de l'institution ne doivent pas favoriser un type de plate-forme plutôt qu'un autre, ni des achats ponctuels massifs. La simplicité dans la gestion des réseaux doit être recherchée. Internet ainsi que les intranets permettent l'utilisation de réseaux hétérogènes qui doivent être préférés aux réseaux "propriétaires» et aux matériels uniques. On doit se garder d'enfermer l'éducation dans une logique unique.

Cependant, l'animation, la gestion et la maintenance des réseaux sont des fonctions très prenantes exigeant la présence de personnels qualifiés. De plus, la multiplication des accès pose le problème des ressources humaines et des coûts d'installation mais aussi celui récurrent des coûts de communication et de maintenance. Malgré tout, des utilisations plus globales des réseaux se font jour, avec un partage des tâches, du travail coopératif, des échanges internationaux (français, langues étrangères mais aussi autres disciplines). Ces nouveaux services autorisent en outre le suivi à distance de l'activité pédagogique dans les académies ou dans les établissements, y compris dans les domaines autres que celui du multimédia éducatif. Ils participent ainsi à l'amélioration d'un dispositif visant à mieux connaître le fonctionnement du système éducatif ainsi que son état d'avancement.

La généralisation de l'utilisation raisonnée du multimédia à l'école n'est pas une mince affaire. Elle exige d'importants efforts, aussi bien collectifs qu'individuels, autant économiques que sociaux: efforts d'équipement adaptés, efforts de formation des personnels, efforts de réflexion sur l'évolution de la nature même de la société, sur ses 
contraintes et ses perspectives, efforts de réflexion sur le rôle et l'organisation de l'ensemble du système éducatif, efforts d'adaptation à de nouvelles formes pédagogiques.

Dans sa mission de formation des futurs citoyens, l'école doit aussi savoir répondre aux exigences nouvelles de la société de l'information, comme elle a su le faire avec la société industrielle. Elle doit jouer pleinement son rôle dans l'évolution des civilisations. Elle seule est en mesure d'offrir et de garantir l'égalité de l'accès aux outils d'information et de communication dans sa mission de formation de citoyens conscients, autonomes et acteurs responsables de la "société de la connaissance». C'est bien dans ce sens que devrait s'inscrire la grande réflexion sur l'école.

\section{RÉSUMÉS}

Pour l'école du XXI ${ }^{\mathrm{e}}$ siècle, l'utilisation des nouvelles technologies est un enjeu majeur qui rentre dans ses missions de formation des futurs citoyens. Elle se doit, en effet, de répondre aux exigences de la société de l'information, comme elle a répondu aux exigences de la société industrielle, même s'il lui en coûte d'importants efforts tant collectifs qu'individuels, économiques que sociaux.

\section{INDEX}

Index géographique : France

\section{AUTEUR}

\section{GUY POUZARD}

Inspecteur général de l'Éducation nationale, ministère de l'Éducation nationale de la Recherche et de la Technologie, Paris, France. 